\title{
Autoritarismo y conciencia de muerte en estudiantes universitarios españoles
}

\section{Authoritarianism and Awareness of Death in Spanish College Students}

\author{
María de la Villa Moral-Jiménez \& Carlos Suárez-Juesas \\ Universidad de Oviedo, Oviedo, España
}

\begin{abstract}
Resumen: El objetivo del presente estudio fue relacionar el autoritarismo y la conciencia de muerte, postulándose que los sujetos con un mayor grado de autoritarismo presentarían más miedo ante la muerte propia y ajena. Mediante un muestreo bietápico de conveniencia con submuestreo aleatorio intragrupo se seleccionaron 202 estudiantes universitarios españoles con edades entre los 18 y los 25 años $(M=20,50 ; D T=2,57)$. Los resultados aportan evidencias empíricas acerca de las diferencias significativas en función del género en el factor Antiintraceptividad de la Escala F, con tasas más elevadas manifestadas en el género masculino y diferencias atribuibles a la edad (factor Poder y fortaleza en la Escala F y Convencionalismo y Sumisión autoritaria en la Escala Autoritarismo de Derechas [RWA, por su sigla en inglés] en los jóvenes de mayor edad respecto del nivel más bajo). En ambas escalas se ha confirmado la existencia de diferencias significativas tanto en función de la clase social como de la orientación política. También se han hallado evidencias empíricas que confirman que las personas autoritarias presentan mayor miedo a la muerte propia y ajena y son más conscientes del proceso de morir. Se discute acerca de las implicaciones de tales hallazgos y se plantean futuras líneas de investigación en la temática.
\end{abstract}

Palabras clave: autoritarismo, conciencia de muerte, Escala F, Escala RWA, estudiantes universitarios, España.
Abstract: The aim of this study was to relate authoritarianism and awareness of death, suggesting that subjects with a higher degree of authoritarianism would present a greater fear of their own and others death. 202 Spanish university students, aged between 18 and 25 years $(M=20,50 ; D T=2,57)$ were selected using a two-stage sampling of convenience with an intra-group random subsample. The results obtained in this study provide empirical evidence on the significant differences by gender in the Anti-intraceptivity factor of F Scale, with higher rates expressed in the male gender and differences due to age (Power and Strength factor in F Scale and Conventionalism, Authoritarian submission in the Right Wing Authoritarianism [RWA] Scale in the older students compared to the lowest level). In both scales it was confirmed that there are significant differences, both in terms of social class and political orientation. It has also been found empirical evidence confirming that the authoritarian people have a greater fear of their own and others death, and are more aware of the dying process. The implications of these findings are discussed and future research lines in the subject are proposed.

Keywords: authoritarianism, awareness of death, F Scale, RWA Scale, university students, Spain.

Contacto: M. V. Moral-Jiménez. Facultad de Psicología. Plaza Feijóo, s/n - Despacho 211. CP: 33003 Oviedo, Principado de Asturias (España). Correo electrónico: mvilla@uniovi.es

Cómo citar: Moral-Jiménez, M. V. \& Suárez-Juesas, C. (2015). Autoritarismo y conciencia de muerte en estudiantes universitarios españoles. Revista de Psicología, 24(2), 1-21.

$\mathrm{http} / / / \mathrm{dx}$. doi.org/10.5354/0719-0581.2015.38402 


\section{Introducción}

El autoritarismo es un tema clásico en el campo de la psicología social que ha sido objeto de estudio desde diversas perspectivas, desde su conceptualización inicial hasta las reinterpretaciones más actuales, así como en función de las diferentes etapas de su evaluación empírica (Martin, 2001; Seoane \& Garzón, 1992; Wolfe, 2005). El estudio del autoritarismo como síndrome de la personalidad sin duda alguna recibió su mayor impulso de la mano de Adorno, Frenkel-Brunswik, Levinson y Sanford (1950) quienes lo definieron como un síndrome mediante el cual la representación social externa del individuo va ligada a una fuerte represión de impulsos, por lo que su actitud hacia la autoridad y hacia sí mismo se encuentra cargada de contenidos irracionales y contradictorios. Esto genera que el individuo logre su propio ajuste social únicamente si encuentra placer en la subordinación y en la obediencia, haciendo que surjan impulsos sadomasoquistas que son condición básica y resultado del ajuste social (Adorno et al., 1950). A su vez, Fromm hacia 1941 daba por iniciada la búsqueda de las raíces psicológicas que sustentaban el autoritarismo, aunando la fuerte tendencia a la sumisión y a la dependencia de otras personas -que es consecuencia de los sentimientos de inferioridad, impotencia e insignificancia individual- y la tendencia a someter a los demás. Como es sabido, el estudio de Adorno et al. (1950) se basaba en estas ideas de Fromm (1941), aunque fundamentaba que la persona autoritaria tiene una personalidad conformista y rígida, que experimenta placer obedeciendo y siendo obedecido, al mismo tiempo que admira la fuerza y rechaza la debilidad, presenta gran identificación con quienes se sitúan más alto en la jerarquía de autoridad. Con la ela- boración de la Escala F se perseguían los objetivos fundamentales de "medir prejuicios y medir tendencias antidemocráticas a nivel de personalidad" (Ovejero, 1982, p. 42).

Ciertamente, el concepto de autoritarismo descrito recibió varias críticas, tanto de tipo teórico como metodológico. Respecto de las primeras, estas se referían a la conceptualización de la persona autoritaria como un tipo de personalidad conservadora, de derechas (Shils, 1954), aunque caracteriza a sujetos de cualquier ideología. De este modo, el desplazamiento del foco de atención hacia el concepto de dogmatismo, por parte de Rokeach (1960) proporcionó una distinción entre las creencias y el contenido mentalidad abierta-cerrada, de tal forma que un individuo puede poseer creencias democráticas, pero defenderlas de modo intolerante o autoritario frente a quienes no las comparten.

Como es bien sabido, en la concepción original del síndrome de autoritarismo los investigadores de Berkeley establecen nueve factores (Adorno, Frenkel-Brunswik, Levinson, \& Sanford, 1969) que representan los principales rasgos expresivos de la personalidad autoritaria, si bien no cubren todas sus características. Las dimensiones exploradas son:

a) Convencionalismo. Adhesión rígida a los valores convencionales de la clase media y disposición a sentirse ansioso ante la violación de dichos valores.

b) Sumisión autoritaria. Actitud de sumisión y aceptación incondicional respecto de las autoridades morales idealizadas del endogrupo. Explora la necesidad exagerada de someterse ante una gran variedad de figuras que representan autoridad y, junto con el convencionalismo, muestra a un individuo orientado 
hacia la presión externa, en vez de hacia el control personal.

c) Agresividad autoritaria. Tendencia a buscar y condenar, rechazar y castigar a los individuos que violan los valores convencionales. Consiste en la gratitud hacia figuras autoritarias de su propio grupo y hostilidad hacia otros grupos ajenos.

d) Antiintraceptividad. Oposición a lo subjetivo, a la autorreflexión, a la introspección. El sujeto es antisubjetivista, ya que teme producir pensamientos equivocados y evita la expresión de sus sentimientos por temor a perder el control emocional, de modo que esta actitud le conduce a una devaluación de lo humano y a una sobrevaloración de lo físico.

e) Superstición y estereotipia. Creencia en la determinación sobrenatural del destino humano e inclinación a pensar en categorías rígidas, lo cual se asocia al hecho de que la persona se aferre a explicaciones primitivas de los eventos humanos y mundanos.

f) Poder y fortaleza. Preocupación por la dimensión dominio-sumisión, fuertedébil, etc. en sus relaciones interpersonales, identificándose con las figuras que representan poder y valorando en exceso la fuerza y la dureza. El individuo admira el poder de los otros y se somete a él, pero rechaza la debilidad que ello implica.

g) Destructividad y cinismo. Hostilidad y vilipendio general de la humanidad, lo cual conduce a una agresividad indiferenciada, alentada con un mínimo de estimulación externa, hacia grupos minoritarios o hacia grupos políticos cuya persecución resulte provechosa.

h) Proyectividad. Proyección de impulsos reprimidos hacia otras personas o even- tos del contexto inmediato. Al proyectar hacia el exterior impulsos emocionales inconscientes, las personas autoritarias tienden a creer que en el mundo suceden cosas desenfrenadas y peligrosas.

i) Sexo. Preocupación exagerada por las cuestiones sexuales.

La definición de autoritarismo con mayor aceptación en la actualidad, según Duckitt y Fisher (2003) es la aportada por Altemeyer $(1981,1996,1998)$ para quien el síndrome de personalidad autoritaria se vincula a una combinación de tres factores actitudinales centrales: Sumisión autoritaria, Agresión autoritaria y Convencionalismo. De este modo, resulta de interés su evaluación del autoritarismo de derechas mediante la Escala de Autoritarismo de Derechas (RWA por su sigla en inglés), que ha sido utilizada en diversas investigaciones (e.g., Ahrens \& Innes, 1994; Duckitt, 2000; Ekehammar, Akrami, Gylje, \& Zakrisson, 2004; Farre \& Duckitt, 1994; McFarland, Ageyev, \& Djintcharadze, 1996; Roccato, Re, \& Sclauzero, 2002; Seoane \& Garzón, 1992; Sidanius \& Pratto, 1999). Asimismo, mediante las versiones abreviadas de la RWA (Schultz \& Stone, 1994; Walter, 1996; Zakrisson, 2005) y la adaptación en población chilena de la versión abreviada realizada en el estudio de Cárdenas y $\mathrm{Pa}$ rra (2010), se ha tratado de subsanar el marcado uso idiosincrásico que atentaría contra su validez (Schaffer \& Riordan, 2003), así como también se discute acerca de la dimensionalidad (Etchezahar, Prado-Gascó, Jaume, \& Brussino, 2014; Funke, 2005; Manganelli, Bobbio, \& Canova, 2007; Smith \& Winter, 2002).

Han sido varios los estudios realizados respecto del autoritarismo y diversas las variables sociodemográficas y actitudinales investigadas. Así, en un estudio 
realizado por Comuni, Langelotti, Jaume, Rodríguez y Etchezahar (2010) se pretendía determinar mediante los resultados obtenidos en la Escala RWA, las diferencias en las puntuaciones en función de la orientación política y de las siguientes variables sociodemográficas: edad, sexo, empleo y etapa de la carrera. En dicho estudio, Comuni et al. (2010) observaron diferencias estadísticamente significativas en cuanto al puntaje total obtenido en la Escala RWA y el posicionamiento ideológico en el que se ubican los sujetos, teniendo una mayor puntuación aquellos orientados hacia la derecha política, aunque no obtuvieron diferencias en función de la edad y se confirma la hipótesis de invarianza de género, tendencia hallada en otras investigaciones (e.g., Cárdenas, Meza, Lagues, \& Yañez, 2010; Etchezahar, 2012; Petit \& Costa, 2011; Silván-Ferrero \& Bustillos, 2007). En este sentido, en el ámbito organizacional Rajan y Krishnan (2002) al evaluar el impacto de género sobre la frecuencia del uso de estrategias de influencia, cantidad de poder y autoritarismo, tampoco hallaron diferencias atribuibles al género. Asimismo, se ha confirmado que el autoritarismo correlaciona negativamente con el nivel cultural y educativo, hallándose un efecto interactivo del género y del tipo de sociedad urbana o rural (Ovejero, 1986, 1987).

A su vez, Duncan, Peterson y Winter (1997) relacionaron el autoritarismo con roles de género, encontrando que altas puntuaciones en el autoritarismo se relacionaron con una identidad de género favorable a roles y actitudes tradicionales, tendencia confirmada en otros estudios (e.g., Canto, Perles, \& San Martín, 2014; Etchezahar et al., 2014; Peterson \& Zurbriggen, 2010; Rubinstein \& Lansisky, 2013). En estudios de corte transcultural como el de Brandt y Henry
(2012) se concluye que las mujeres respaldaron más los valores autoritarios que los hombres, especialmente en sociedades individualistas con altos niveles de desigualdad de género, aunque los países más colectivistas no presentan esta relación desigualdad-autoritarismo.

Por otro lado, también se ha explorado la relación de otros instrumentos diseñados para la medición del autoritarismo, como la Escala "directiveness" de Ray (1976), con las actitudes sociopolíticas como en el estudio de Seoane, Arce y Sabucedo (1988). Al igual que en estudios clásicos (e.g., Shils, 1954) se sigue confirmando la tendencia de que los sujetos que se identifican con una orientación política de derechas respecto de los de izquierdas (Cárdenas \& Parra, 2010) presenten mayor grado de autoritarismo, así como una alta orientación de dominancia social y moralidad conservadora (Jackson \& Gaertner, 2010; Leidner, Castano, Zaizer, \& GinerSorolla, 2010). Asimismo, la relación entre rasgos psicológicos autoritarios y sistemas cognitivos dogmáticos por un lado, y distintas actitudes sociopolíticas por otro, también ha sido objeto de estudio, de manera que se confirma que el autoritarismodogmatismo es un potente predictor de las actitudes y preferencias sociopolíticas (Temkin \& Flores-Ivich, 2011).

En todo caso, aunque el autoritarismo de derechas y la orientación de dominación son buenos predictores del prejuicio y de la personalidad autoritaria, se constata una relación diferencial con las actitudes sociopolíticas, ya que según Duriez, Van Hiel y Kossowska (2005), mientras que el prejuicio basado en el autoritarismo de derechas es motivado por el miedo y los sentimientos de amenaza, el activado por la dominancia social se vincula a los modelos de lucha por los recursos y el poder. También resultan de sumo interés los es- 
tudios referidos a actitudes relevantes para el posicionamiento ideológico y la estructura social, como el de Carvacho y Haye (2008), en el que se concluye que la relación entre autoritarismo y nivel educacional está condicionada por la clase social. Asimismo, se ha evaluado la relación predictiva entre el autoritarismo y la dominancia social con el prejuicio étnico y actitudes sexistas, como en el estudio de Díaz-Lázaro y Toro (2014), resultando el autoritarismo la variable con mayor influencia en la varianza de los distintos tipos de prejuicios (Ungaretti, Etchezahar, \& Brussino, 2015).

Dado que el interés del estudio se centró en la relación entre autoritarismo y conciencia de muerte, es preciso fundamentar el segundo constructo que encuentra apoyo en la Teoría de la Manipulación del Terror (TMT), resultado de la colaboración entre Greenberg, Pyszczynski y Solomon (1986). Esta teoría parte de la formulación de Becker (1973) y sostiene que el resaltar la condición mortal genera las siguientes condiciones: a) la negación sincera, que ocurre al enfrentarnos a la amenaza de muerte, y consiste en negar la evidencia y va acompañada de un desprecio ante esta idea y b) el intento de mantener la autoestima, la defensa de los propios valores y visión del mundo, lo que se identifica con una defensa más estable (Pyszczynski, Gutenberg, \& Solomon, 1999). Estos mecanismos de defensa, protectores de la ansiedad empiezan a actuar inmediatamente después de la amenaza y pueden proporcionar la clave de la personalidad autoritaria. Implica desprecio por otras creencias, lo que puede generar prejuicios, discriminación e incluso actos agresivos (Greenberg et al., 1986; Greenberg, Simon, Pyszczynski, Solomon, \& Chatel, 1992). Así, en un estudio realizado por Rosenblatt, Greenberg, Solomon, Pyszczynski y Lyon (1989) se demostró que las personas autoritarias se aferran a su concepción cultural, las formas correctas de comportarse, los principios religiosos y/o la moralidad convencional. En este sentido, de acuerdo con Stone (2001), la personalidad autoritaria es particularmente sensible a la amenaza de la muerte, de manera que la conciencia de muerte afecta a los autoritarios, ya que las concepciones alternativas de la realidad que amenazan sus creencias pueden provocarles recelos al poner en evidencia sus rechazos a otras visiones (Etchezahar \& Brussino, 2015). Por el contrario, un recurso protector de la ansiedad existencial es la autoestima, al considerarse que una autoestima alta implica tener una gran confianza y valoración, por lo tanto si las normas del autoritario realmente son estrictas y rígidas, su autoestima será más difícil de mantener (Bun, Louiselle, Misukanis, \& Mueller, 1988).

Al inducir la conciencia de muerte aparecen dos conductas propias de la personalidad autoritaria, tal y como recoge Stone (2001): el carácter punitivo y el etnocentrismo. Respecto del carácter punitivo, la investigación de Rosenblatt et al. (1989) señala que se les pidió a unos jueces que establecieran un castigo a una supuesta prostituta acusada, provocándoles la conciencia de muerte a unos jueces y a otros no, siendo mayor la severidad en el castigo potencial de los primeros respecto del grupo control (recogido en Greenberg, Solomon, \& Pyszczynski, 1997). Por otro lado, resulta de interés el etnocentrismo, descrito como una tendencia emocional que hace de la cultura propia el criterio exclusivo para interpretar los comportamientos de otros grupos, razas o sociedades. Así, Greenberg et al. (1990) hallaron que, bajo condiciones de conciencia de muerte, los autoritarios altos eran mucho más negativos hacia sujetos valorados como distintos a ellos, que los que presen- 
taban bajo autoritarismo, lo cual es indicativo de su particular susceptibilidad a los pensamientos de muerte. En concreto, observaron que personas cristianas, bajo condiciones de conciencia de muerte, mostraron una discriminación positiva hacia otras personas cristianas y una discriminación negativa ante personas judías. Esto confirma que los autoritarios normalmente están a favor de su propio grupo y discriminan a los miembros de grupos ajenos, de acuerdo con Duckitt (1989).

En la literatura especializada acerca del tema destacan varios estudios en los que se explora la respuesta de las personas autoritarias a la conciencia de muerte. Así, Burling (1993) observó que la conciencia de la muerte aumenta la preocupación por la posición social y por la necesidad de éxito simbólico. Por su parte, en el metaanálisis emprendido por Jost, Glaser, Kruglanski y Sulloway (2003) entre las variables que mejor predicen la adhesión a valores y opiniones políticamente conservadoras, se ha hallado que el miedo a la muerte o saliencia (accesibilidad, prominencia, notabilidad, recuerdo) de la mortalidad ha mostrado una relación consistente e intensa. En este sentido, en relación con la orientación política, Espinosa y Calderón (2006) ratifican que los sujetos que presentan más miedo a la muerte son los de orientación política de centro derecha/derecha, confirmando que el miedo a la muerte podría incrementar el sesgo endogrupal en estos sujetos, hecho que se asocia al hallazgo de que la saliencia de la mortalidad es mayor en personas que comparten valores colectivistas autoritarios (Solomon, Greenberg, \& Pyszczynski, 1991). En esta misma dirección, se confirma que las personas autoritarias manifiestan mayor miedo o ansiedad ante la muerte y presentan comportamientos temerosos asociados en ma- yor medida a ideologías conservadoras o de derechas, lo cual se encuentra refrendado en estudios acerca del manejo del terror y amenazas existenciales (Piñuela, 2015).

Fundamentando lo anterior, el principal objetivo del presente estudio fue relacionar el autoritarismo y la conciencia de la muerte en una muestra de estudiantes universitarios, postulándose que aquellos alumnos con un mayor grado de autoritarismo presentarían mayor miedo o ansiedad ante la muerte. Se procedió al análisis de las diferencias en personalidad autoritaria en función de una serie de variables sociodemográficas, tales como género y edad, postulándose que los varones de mayor nivel de edad puntuarían más en los factores que exploran este constructo. Asimismo, la investigación se planteó como objetivo analizar las diferencias en personalidad autoritaria en función de variables actitudinales, tales como la orientación política, en el sentido de que los sujetos que se autoperciben como de derechas presentarían puntuaciones más elevadas.

\section{Método}

\section{Participantes}

Se obtuvo una muestra de 202 estudiantes (25,7\% hombres; $74,3 \%$ mujeres) compuesta por estudiantes de la Universidad de Oviedo con edades comprendidas entre los 18 y $\operatorname{los} 25$ años $(M=20,50 ; D T=$ $2,57)$. Los participantes fueron reclutados entre estudiantes de primer y tercer curso de universidad de los grados de Psicología y Turismo, seleccionados mediante un muestreo bietápico de conveniencia de modo incidental, condicionado por los vínculos de docencia e investigación con submuestreo aleatorio intragrupo. 


\section{Variables investigadas e instrumentos}

Para evaluar el autoritarismo se ha empleado la Escala $F$ en su versión 45/40 validada por Adorno et al. (1969) y que presenta adecuados índices de confiabilidad. Consta de 30 ítems evaluados mediante la Escala Likert con valores de 1 a $6(1=$ Totalmente en desacuerdo a $6=$ Totalmente de acuerdo). En el estudio se ha obtenido un $\alpha$ global de Cronbach de ,920 e indicadores de confiabilidad con valores que oscilan entre el ,808 hallado en la dimensión Agresividad autoritaria y ,657 en Destructividad y cinismo. Asimismo, en los análisis correlacionales se obtuvieron valores elevados entre las dimensiones exploradas, tales como entre Agresividad autoritaria y Sexo $(r=, 772)$, Proyectividad y Sumisión y estereotipia $(r=, 779)$, Poder y fortaleza y Sumisión autoritaria $(r=, 722)$, Convencionalismo y Antiintraceptividad $(r=, 688)$, por citar algunos ejemplos.

También se ha aplicado la Escala RWA en una versión reducida, con 12 ítems. Se trata de una escala adaptada que posee ítems más cortos, con una redacción más moderada de los ítems, menos referencias a grupos específicos y que ha sido validada al castellano por Cárdenas y Parra (2010). Mide la covariación de tres grupos de actitudes explicados con anterioridad, la Sumisión autoritaria, Agresión autoritaria y el Convencionalismo. Se emplea la Escala Likert con valores de 1 a $6(1=$ Totalmente en desacuerdo a $6=$ Totalmente de acuerdo). La confiabilidad global de la escala es este estudio es de ,874 ( $\alpha$ de Cronbach) con valores que oscilan entre ,702 (Agresividad autoritaria) y ,635 (Convencionalismo) y también se han hallado valores adecuados de correlación entre los factores de Convencionalismo y Agresividad autoritaria $(r=$ ,608), Sumisión autoritaria y Agresividad autoritaria $(r=, 564)$ y Convencionalismo y Sumisión autoritaria $(r=, 523)$.

En cuanto a la Escala de Miedo a la Muerte (EMMCL) de Collett-Lester (1969) se ha empleado la versión validada al castellano (Espinoza, Sanhueza, \& Barriga, 2011; Tomás-Sábado, Limonero, \& Abdel-Khalek, 2007). Consta de 28 ítems evaluados mediante Escala Likert de cinco puntos con valores de 1 a $5(1=N a d a$ a $5=$ Mucho) y cuatro subescalas que proporcionan información multidimensional acerca del "miedo a la propia muerte", "el miedo al proceso de morir propio", "miedo a la muerte de otros" y "miedo al proceso de morir de otros". Se obtiene un puntaje por cada subdimensión, promediando las respuestas respectivamente. Las puntuaciones promedio más altas indican mayor miedo a la muerte o al proceso de morir. El $\alpha$ de Cronbach hallado en este estudio es de, 927 , con valores que oscilan entre ,802 (miedo a la propia muerte) y ,716 (miedo al proceso de morir de otros) y una adecuada correlación interelementos como entre la dimensión "miedo al proceso de morir propio" y "miedo a la propia muerte" $(r=, 729)$ o entre "miedo al proceso de morir de otros" y "miedo a la propia de otros" ( $r=$ ,683).

Asimismo, se han realizado preguntas respecto de características sociodemográficas como la edad, el género y el nivel de estudios. También se han incluido sendas preguntas para que los participantes reporten la clase social (evaluada con cuatro opciones de respuesta desde "muy baja" a "muy alta") y el posicionamiento político autopercibido (con cinco opciones de respuesta desde "extrema izquierda" hasta "extrema derecha") con el objetivo de determinar posibles diferencias en función de estas variables. En ambos casos se ha recodificado la opción de respuesta de 
clase social "muy alta" como "alta" y orientación política de "extrema derecha" como "derecha", dado que únicamente un sujeto se ha identificado con estos niveles de respuesta.

\section{Procedimiento y análisis de datos}

Los participantes fueron reclutados como voluntarios y respondieron las escalas de forma anónima con garantías de confidencialidad, siendo informados de los fines de la investigación y del uso exclusivo de los resultados para tales fines. Los test fueron respondidos en horario de clases, de modo que la aplicación fue grupal y en sus respectivas aulas, disponiendo de un tiempo aproximado de 30 minutos y contando con la participación de personal investigador.

El análisis de los datos se realizó mediante el paquete estadístico SPSS 18.0.3. Se realizaron comparaciones de medias, correlaciones bivariadas y la evaluación de la consistencia interna. Al no cumplirse los criterios de homocedasticidad, se realizaron comparaciones entre rangos de medias con las pruebas no paramétricas de Kruskal-Wallis y MannWhitney. En este caso, la varianza de error de las variables no es constante y el valor predicho no se corresponde con la media de la variable a predecir. En los datos su incumplimiento conduce a suponer heterocedasticidad, fenómeno frecuente en datos de corte transversal y que puede interpretarse apelando a la posible omisión de variables importantes dentro del modelo a estimar, ya que si se omite una variable de relevancia en la especificación, esta quedará parcialmente recogida dentro de las perturbaciones aleatorias. La Prueba de Levene ha resultado significativa con un valor $<, 05$ con lo cual se recomienda el empleo de prue- bas generalizadas (pruebas no paramétricas), concluyéndose que hay una diferencia entre las variaciones en la población. Por tanto, se ha optado por este tipo de análisis, debido al cumplimiento del criterio de heterocedasticidad, y teniendo en cuenta los tamaños muestrales distintos, para evitar el cálculo incorrecto de la varianza y de parámetros ineficientes, así como ante la posible invalidación de los contrastes de significación.

\section{Resultados}

En primer lugar, al evaluar el autoritarismo mediante la Escala $\mathrm{F}$ se hallaron diferencias en función de las variables género y edad. Empleando la prueba no paramétrica de Mann-Whitney se obtuvieron diferencias intergénero en el factor Antiintraceptividad $(Z=-2,56, p=$ $, 01)$, siendo mayor el grado de autoritarismo en el género masculino. En cuanto a la variable edad, mediante la Prueba Kruskal-Wallis resulta significativo el factor Poder y fortaleza $\left(\chi^{2}(2)=8,99, p=\right.$ $, 01)$, confirmándose mayor grado de autoritarismo en los jóvenes de mayor edad (23 años o más) respecto del grupo de 18 y 19 años $(Z=-2,77, p=, 006)$ (véase tabla 1).

Tal y como se recoge en la tabla 2 , en el caso de la Escala RWA no se han hallado diferencias intergénero, aunque sí en función del nivel de edad en los factores Convencionalismo $\left(\chi^{2}(2)=11,42, p=\right.$ ,003) y Sumisión autoritaria $\left(\chi^{2}(2)=\right.$ $7,49, p=, 024)$. En ambos factores se confirma la tendencia predicha, presentando valores más elevados en ambos factores $((Z=-3,06, p=, 002)$ y $(Z=-2,66, p=$ ,008), respectivamente) en los jóvenes de mayor edad respecto de los del nivel 1 (18-19 años). 
Tabla 1

Descriptivos de los factores de la Escala F por género y edad

\begin{tabular}{|c|c|c|c|c|c|}
\hline \multirow[b]{2}{*}{ Escala F } & \multicolumn{2}{|c|}{ Género } & \multicolumn{3}{|c|}{ Nivel de edad } \\
\hline & $\begin{array}{l}\text { Masculino } \\
M(D T)\end{array}$ & $\begin{array}{l}\text { Femenino } \\
\qquad M(D T)\end{array}$ & $\begin{array}{c}\text { 18-19 años } \\
M(D T)\end{array}$ & $\begin{array}{c}\text { 20-22 años } \\
M(D T)\end{array}$ & $\begin{array}{c}23 \text { años o } \\
\text { más } \\
M(D T)\end{array}$ \\
\hline Convencionalismo & ,42(,11) & ,42(,10) & ,41(,10) & ,42(,10) & ,45(,11) \\
\hline Sumisión autoritaria &, $80(, 21)$ &, $83(, 16)$ &, $80(, 15)$ &, $83(, 18)$ &, $85(, 22)$ \\
\hline $\begin{array}{l}\text { Agresividad } \\
\text { autoritaria }\end{array}$ &, $77(, 22)$ & ,75(,19) &, $74(, 18)$ &, $76(, 20)$ &, $79(, 25)$ \\
\hline Antiintraceptividad & ,29(,09) &, $25(, 09) *$ &, $25(, 07)$ & ,27(,09) &, $29(, 10)$ \\
\hline $\begin{array}{l}\text { Superstición y estereo- } \\
\text { tipia }\end{array}$ & ,64(,18) &, $65(, 18)$ & ,66(,19) & ,63(,17) & ,66(,18) \\
\hline Poder y fortaleza &, $58(, 16)$ &, $58(, 15)$ &, $55(, 13)$ &, $58(, 15)$ &, $64(, 20)^{*}$ \\
\hline $\begin{array}{l}\text { Destructividad y ci- } \\
\text { nismo }\end{array}$ &, $17(, 07)$ &, $17(, 06)$ &, $16(, 05)$ &, $17(, 06)$ & ,19(,08) \\
\hline Proyectividad &, $45(, 14)$ & ,44(,16) &, $43(, 18)$ & ,44(,13) &, $45(, 14)$ \\
\hline Sexo & ,29(,10) & ,27(,09) &, $27(, 08)$ &, $28(, 10)$ & ,29(,11) \\
\hline
\end{tabular}

Nota: $M=$ Media, $D T=$ Desviación típica.

$* p<, 01$.

Tabla 2

Descriptivos de los factores de la Escala RWA por género y edad

\begin{tabular}{l|cc|ccc}
\hline \multirow{2}{*}{ Escala RWA } & \multicolumn{3}{|c|}{ Género } & \multicolumn{3}{c}{ Nivel de edad } \\
& Masculino & Femenino & $18-19$ años & $20-22$ años & 23 años o más \\
& $M(D T)$ & $M(D T)$ & $M(D T)$ & $M(D T)$ & $M(D T)$ \\
\hline Convencionalismo & $0,94(0,32)$ & $0,89(0,29)$ & $0,71(0,21)$ & $0,79(0,21)$ & $0,88(0,21)^{*}$ \\
Sumisión autoritaria & $1,36(0,42)$ & $1,29(0,33)$ & $0,85(0,29)$ & $0,91(0,30)$ & $1,05(0,28)^{*}$ \\
Agresividad & $0,78(0,22)$ & $0,77(0,22)$ & $1,27(0,33)$ & $1,31(0,37)$ & $1,39(0,38)$ \\
autoritaria & & & & & \\
\hline
\end{tabular}

Nota: $M=$ Media, $D T=$ Desviación típica.

$* p<, 01$.

Se ha comprobado que todos los factores de la Escala F muestran diferencias significativas en función la clase social: Convencionalismo $\left(\chi^{2}(2)=7,97, p=, 02\right)$, Sumisión autoritaria $\left(\chi^{2}(2)=11,50, p=, 003\right)$, Agresividad autoritaria $\left(\chi^{2}(2)=8,87, p=, 012\right)$, Antiintraceptividad $\left(\chi^{2}(2)=8,05, p=, 02\right)$, Superstición y estereotipia $\left(\chi^{2}(2)=13,51, p\right.$ $=, 001)$, Poder y fortaleza $\left(\chi^{2}(2)=9,54, p=\right.$
,008), Destructividad y cinismo $\left(\chi^{2}(2)=\right.$ $8,90, p=, 012)$, Proyectividad $\left(\chi^{2}(2)=\right.$ $13,65, p=, 001)$ y $\operatorname{Sexo}\left(\chi^{2}(2)=11,40, p=\right.$ ,003). Se comprobó que los sujetos de clase social alta puntúan más alto en todos los factores anteriormente citados.

Respecto de la orientación política, se siguió el mismo procedimiento y se obtuvo que en 
la Escala $\mathrm{F}$ todos los factores presentan diferencias significativas: Convencionalismo $\left(\chi^{2}(3)=21,16, p<, 001\right)$, Sumisión autoritaria $\left(\chi^{2}(3)=37,79, p<, 001\right)$, Agresividad autoritaria $\left(\chi^{2}(3)=54,06, p<, 001\right)$, Antiintraceptividad $\left(\chi^{2}(3)=16,17, p=, 001\right), \mathrm{Su}-$ perstición y estereotipia $\left(\chi^{2}(3)=47,40, p<\right.$ $, 001)$, Poder y fortaleza $\left(\chi^{2}(3)=27,62, p<\right.$ $, 001)$, Destructividad y cinismo $\left(\chi^{2}(3)=\right.$ 20,36, $p<, 001)$, Proyectividad $\left(\chi^{2}(3)=\right.$ $28,25, p<, 001)$ y $\operatorname{Sexo}\left(\chi^{2}(3)=43,37, p<\right.$ ,001). A la hora de buscar la dirección de estas diferencias, se obtiene que los estudiantes de derechas tienen una puntuación mayor en todos los factores: Convencionalismo $(Z=-4,62, p<, 001)$, Sumisión autoritaria $(Z=-6,02, p<, 001)$, Agresividad autoritaria $(Z=-6,71, p<, 001)$, Antiintraceptividad $(Z=-3,68, p<, 001)$, Superstición y estereotipia $(Z=-6,50, p<001)$, Poder y fortaleza $(Z=-4,98, p<, 001)$, Destructividad y cinismo $(Z=4,47, p<$ ,001), Proyectividad $(Z=-5,10, p<, 001) \mathrm{y}$ Sexo $(Z=-6,20, p<, 001)$ (véase tabla 3$)$.
Seguidamente se realizaron las pruebas no paramétricas sobre la variable clase social y orientación política en este caso en las puntuaciones obtenidas en la Escala RWA. En la clase social se encontró que el factor Convencionalismo $\left(\chi^{2}(2)=6,54, p=, 040\right)$ $\mathrm{y}$ el factor Agresividad autoritaria $\left(\chi^{2}(2)=\right.$ $10,71, p=, 005)$ muestran diferencias significativas. Al investigar la dirección de las diferencias con Mann-Whitney se aprecia que en ambos casos se presentan diferencias entre las clases sociales bajas y altas, obteniendo una mayor puntuación los estudiantes de clase alta.

Realizando el mismo procedimiento con la orientación política en la Escala RWA, aparece que los tres factores de la Escala RWA muestran diferencias significativas entre ellos en función de la orientación política (véase tabla 4). El factor Convencionalismo $\left(\chi^{2}(3)=25,04, p<, 001\right)$, Sumisión autoritaria $\left(\chi^{2}(3)=52,94, p<, 001\right)$, Agresividad autoritaria $\left(\chi^{2}(3)=48,79, p<, 001\right)$.

Tabla 3

Descriptivos en la Escala F en función de la clase social y la orientación política

\begin{tabular}{|c|c|c|c|c|c|c|c|}
\hline \multirow[b]{2}{*}{ Escala F } & \multicolumn{3}{|c|}{ Clase social } & \multicolumn{4}{|c|}{ Orientación política } \\
\hline & $\begin{array}{c}\text { Baja } \\
M(D T)\end{array}$ & $\begin{array}{l}\text { Media } \\
M(D T)\end{array}$ & $\begin{array}{c}\text { Alta / } \\
\text { Muy alta } \\
M(D T)\end{array}$ & $\begin{array}{c}\text { Extrema } \\
\text { izquierda } \\
M(D T)\end{array}$ & $\begin{array}{l}\text { Izquierda } \\
M(D T)\end{array}$ & $\begin{array}{l}\text { Centro } \\
M(D T)\end{array}$ & $\begin{array}{c}\text { Derecha/Extrema } \\
\text { derecha } \\
M(D T)\end{array}$ \\
\hline Convencionalismo &, $36(, 06)$ &, $42(, 10)$ &, $49(, 10) * * *$ &, $41(, 12)$ &, $38(, 10)$ &, $43(10)$ &, $47(, 08)^{*}$ \\
\hline $\begin{array}{l}\text { Sumisión } \\
\text { autoritaria }\end{array}$ &, $75(, 21)$ &, $81(, 17)$ &, $97(, 17)^{* *}$ &, $76(, 25)$ &, $75(, 17)$ &, $83(, 15)$ &, $95(, 12)^{*}$ \\
\hline $\begin{array}{l}\text { Agresividad } \\
\text { autoritaria }\end{array}$ &, $62(, 21)$ &, $75(, 19)$ &, $93(, 24) * * *$ &, $67(, 24)$ &, $64(, 18)$ &, $78(, 17)$ &, $92(, 14)^{*}$ \\
\hline Antiintraceptividad & ,23(,08) & ,26(,09) &, $33(, 08)^{*}$ &, $30(, 05)$ &, $24(, 08)$ & ,26(,09) &, $30(, 08)^{*}$ \\
\hline $\begin{array}{l}\text { Superstición y } \\
\text { estereotipia }\end{array}$ &, $60(, 17)$ &, $64(, 18)$ &, $81(, 17)^{*}$ &, $58(, 14)$ &, $59(, 20)$ &, $65(, 16)$ &, $78(, 11)^{*}$ \\
\hline Poder y fortaleza &, $57(, 15)$ &, $57(, 15)$ &, $74(, 16)^{* *}$ &, $53(, 26)$ &, $53(, 14)$ &, $58(, 13)$ &, $69(, 15)^{*}$ \\
\hline $\begin{array}{l}\text { Destructividad y } \\
\text { cinismo }\end{array}$ &, $12(, 04)$ &, $17(, 06)$ &, $21(, 06) * * *$ &, $15(, 08)$ &, $15(, 05)$ &, $17(, 06)$ &, $21(, 06)^{*}$ \\
\hline Proyectividad &, $38(, 20)$ &, $43(, 12)$ &, $57(, 11)^{*}$ &, $40(, 17)$ &, $40(, 18)$ &, $44(, 11)$ &, $52(, 12)^{*}$ \\
\hline Sexo &, $18(, 07)$ & ,27(,09) &, $35(, 11)^{* *}$ &, $26(, 11)$ &, $23(, 08)$ &, $29(, 09)$ &, $36(, 07)^{*}$ \\
\hline
\end{tabular}

Nota: $* p<, 001 ; * * p<, 01 ; * * * p<, 05$. 
Tabla 4

Descriptivos en la Escala RWA en función de la clase social y la orientación política

\begin{tabular}{l|ccc|cccc}
\hline \multirow{2}{*}{ Escala RWA } & Baja & $\begin{array}{c}\text { Clase social } \\
\text { Media }\end{array}$ & $\begin{array}{l}\text { Clase social } \\
\text { Alta/Muy alta }\end{array}$ & $\begin{array}{c}\text { Extrema } \\
\text { izquierda }\end{array}$ & $\begin{array}{c}\text { Orientación política } \\
\text { Izquierda }\end{array}$ & $\begin{array}{c}\text { Centro } \\
\text { Derecha } \\
/ \text { Extrema } \\
\text { derecha }\end{array}$ \\
& $M(D T)$ & $M(D T)$ & $M(D T)$ & $M(D T)$ & $M(D T)$ & $M(D T)$ & $M(D T)$ \\
\hline $\begin{array}{l}\text { Convencio- } \\
\text { nalismo }\end{array}$ & $0,57(0,15)$ & $0,77(0,21)$ & $0,84(0,26)^{* * *}$ & $0,67(0,36)$ & $0,71(0,21)$ & $0,76(0,19)$ & $0,92(0,19)^{*}$ \\
$\begin{array}{l}\text { Sumisión } \\
\text { autoritaria }\end{array}$ & $0,73(0,15)$ & $0,90(0,30)$ & $1,06(0,33)$ & $0,57(0,32)$ & $0,71(0,27)$ & $0,95(0,27)$ & $1,13(0,19)^{*}$ \\
$\begin{array}{l}\text { Agresividad } \\
\text { autoritaria }\end{array}$ & $1,15(0,24)$ & $1,29(0,35)$ & $1,66(0,39)^{* *}$ & $1,04(0,31)$ & $1,14(0,31)$ & $1,34(0,33)$ & $1,60(0,28)^{*}$ \\
\hline
\end{tabular}

Nota: $* p<, 001 ; * * p<, 01 ; * * * p<, 05$.

En los tres factores las diferencias se dan en la dirección de los estudiantes de derechas, ya que estos tienen una mayor puntuación en los factores, Convencionalismo $(Z=-$ $4,63, p<, 001)$, Sumisión autoritaria $(Z=-$ $6,22, p<, 001)$ y Agresividad autoritaria $(Z$ $=-6,37, p<, 001)$.

Una vez encontradas las diferencias en las puntuaciones de las escalas de autoritarismo en función de las variables sociodemográficas, se hallaron las diferencias en el grado de conciencia de la muerte en función del grado de autoritarismo. Para ello se recurrió a las pruebas no paramétricas usadas con anterioridad. Así, respecto de los grados de autoritarismo explorados mediante la Escala $\mathrm{F}$, aparece que dos factores muestran diferencias significativas: Miedo a la propia muerte $\left(\chi^{2}(2)=7,0, p=, 03\right)$ y Miedo a la muerte de otros $\left(\chi^{2}(2)=\right.$ 7,93, $p=, 02)$. Al hallar la dirección en la que se dan las diferencias mediante Mann-Whitney, se aprecia que tanto el factor Miedo a la propia muerte $(Z=-$ $2,61, p=, 009)$ como el factor Miedo a la muerte de otros $(Z=-2,19, p=, 03)$ muestran una mayor puntuación para los sujetos con un alto grado de autoritarismo respecto de los que presentan valores más bajos.

Finalmente, atendiendo a las diferencias en las puntuaciones en la EMMCL en función de las puntuaciones en la Escala RWA, todos los factores de la Escala Collett-Lester muestran diferencias en sus puntuaciones en función del grado de autoritarismo diferenciado con la Escala RWA (véase tabla 5). Esta tendencia se cumple en el factor Miedo a la propia muerte $\left(\chi^{2}(2)=11,75, p=, 003\right)$, Miedo al propio proceso de morir $\left(\chi^{2}(2)=6,18\right.$, $p=, 045)$, Miedo a la muerte de otros $\left(\chi^{2}(2)=10,76, p=, 005\right)$ y Miedo al proceso de morir de otros $\left(\chi^{2}(2)=11,21, p=\right.$ ,004). Se confirma la tendencia explicitada tanto en el factor Miedo a la propia muerte $(Z=-3,38, p=, 001)$, como el resto de factores, Miedo al propio proceso de morir $(Z=-2,58, p=, 01)$, Miedo a la muerte de otros $(Z=-3,05, p=, 002)$ y Miedo al proceso de morir de otros $(Z=-$ $3,26, p=, 001)$, en el sentido de que los estudiantes con un alto grado de autoritarismo presentan mayor conciencia de muerte propia y ajena. 
Tabla 5

Descriptivos en la Escala Collett-Lester en función de los grados de autoritarismo (Escala F y Escala RWA)

\begin{tabular}{l|ccc|ccc}
\hline \multirow{2}{*}{$\begin{array}{l}\text { Escala F/ Escala } \\
\text { RWA }\end{array}$} & \multicolumn{3}{c|}{ Autoritarismo Escala F } & \multicolumn{3}{c}{ Autoritarismo Escala RWA } \\
& Bajo & Medio & Alto & Bajo & Medio & Alto \\
& $M(D T)$ & $M(D T)$ & $M(D T)$ & $M(D T)$ & $M(D T)$ & $M(D T)$ \\
\hline $\begin{array}{l}\text { Miedo a la } \\
\text { propia muerte }\end{array}$ & $0,66(0,19)$ & $0,76(0,24)$ & $0,80(0,23)^{* *}$ & $0,64(0,22)$ & $0,74(0,24)$ & $0,86(0,20)^{*}$ \\
$\begin{array}{l}\text { Miedo al propio } \\
\text { proceso de morir }\end{array}$ & $1,86(0,54)$ & $1,92(0,50)$ & $2,07(0,53)$ & $1,77(0,52)$ & $1,93(0,51)$ & $2,13(0,38)^{* *}$ \\
$\begin{array}{l}\text { Miedo a la } \\
\text { muerte de otros }\end{array}$ & $2,11(0,54)$ & $2,14(0,44)$ & $2,41(0,38)^{* *}$ & $1,91(0,53)$ & $2,19(0,44)$ & $2,32(0,33)^{*}$ \\
$\begin{array}{l}\text { Miedo al proce- } \\
\text { so de morir de } \\
\text { otros }\end{array}$ & $1,90(0,51)$ & $2,00(0,44)$ & $2,13(0,50)$ & $1,79(0,48)$ & $2,00(0,46)$ & $2,20(0,34)^{*}$ \\
\hline
\end{tabular}

Nota: Autoritarismo bajo: <2,30; Autoritarismo medio: 2,30-3,70; Autoritarismo alto: > 3,70.

$* p<, 01 ; * * p<, 05$.

\section{Discusión}

La vinculación que se ha propuesto en este estudio entre autoritarismo y conciencia de muerte apoya los hallazgos relativos a que las personas autoritarias reportan mayor miedo ante la muerte y presentan comportamientos temerosos asociados en mayor medida a ideologías conservadoras, en el mismo sentido que los hallazgos confirmados por Stone (2001). Asimismo, los resultados obtenidos en este estudio aportan evidencias empíricas respecto de las diferencias en los grados de conducta autoritaria en función de variables tales como género, edad, clase social y orientación política, lo cual refrenda otros hallazgos recogidos en la literatura acerca del tema (Cárdenas et al., 2010; Comuni et al., 2010; Etchezahar, 2012; Petit \& Costa, 2011). Si bien no se han obtenido diferencias intergénero en autoritarismo, se han confirmado mayores tasas de autoritarismo en estudiantes pertenecientes a la clase alta y con una orientación política de derechas. En concreto, en la medición del autoritarismo se han hallado diferencias en el factor Antiintraceptividad de la Escala F con tasas más elevadas manifestadas en el género masculino y diferencias atribuibles a la edad (factor Poder y fortaleza en la Escala F y Convencionalismo y Sumisión autoritaria en la Escala RWA en los jóvenes de mayor edad respecto al nivel más bajo).

En los factores evaluados en ambas escalas se ha confirmado la existencia de diferencias significativas tanto en función de la clase social (a excepción del factor Sumisión autoritaria) como de la orientación política (McClosky \& Chong, 1985). A este respecto, el carácter novedoso del hallazgo de que los estudiantes de clase social alta puntúen más en Autoritarismo puede deberse a que en estos sujetos la potencial amenaza ante el cambio es mayor y podría conducirles a un deseo manifiesto de mantener las convenciones, valores sociales y actitudes ideológicas inalterados, preservándolos de los cambios que se están produciendo en países como España en los últimos años a raíz de la crisis deprivativa que se está sufriendo 
(Sirvent, 2013) y de la fracturación y/o desmantelamiento del calificado Estado del bienestar, con el incremento de las desigualdades y la pobreza. El autoritarismo bajo la forma de intolerancia y cierta pasividad representan valores característicos de algunos sectores de la actual sociedad española, asociados a bajos niveles de confianza interpersonal que se relacionan con el predominio de valores autoritarios en condiciones como las actuales de crisis, desigualdad y desesperanza (Sarrión, 2015; Velázquez-Gaztelu, 2015; Wolf, 2015) que afecta especialmente a los jóvenes que se enfrentan a condiciones de vulnerabilidad (Hernández \& López, 2015).

De este modo, en futuras investigaciones se considerará que este efecto de la clase social debe ser indagado con mayor detenimiento, asociando la configuración ideológica de los sujetos a factores estructurales de la sociedad. En todo caso, otra explicación tentativa puede estar asociada a errores de selección muestral y/o a la modalidad de evaluar la adscripción a la clase social mediante la autopercepción de los participantes, lo cual podría haberse subsanado empleando indicadores no percibidos, sino más objetivos, tales como el nivel socioeconómico.

También se han hallado evidencias empíricas que confirman que las personas autoritarias presentan mayor miedo a la muerte, lo cual es refrendado en las investigaciones previas de Greenberg et al. (1990) y Burling (1993), donde se obtuvo que son particularmente más susceptibles a los pensamientos de muerte. Dado que las personas autoritarias tienen concepciones, creencias y valores mucho más rígidos, cualquier intento de violación de dichos aspectos tendrá una mayor repercusión. Se concuerda con Stone (2001) en que estos valores y creencias tan estrictos se asocian a una menor autoestima, que actúa como elemento regulador de la defensa estable ante la conciencia de muerte, lo cual se asocia a la potenciación de mecanismos de defensa que generan una mayor ansiedad ante la potencial amenaza (Stone, 2001). A su vez, a partir del metaanálisis emprendido por Jost et al. (2003) se concluye que los autoritarios no es que presenten menor autoestima, sino que responden a la amenaza de la muerte de manera diferente, de modo que la motivación activada por el miedo y la tendencia a percibir el mundo como potencialmente peligroso, son características básicas de las personas autoritarias. En este sentido, se ha confirmado que la conciencia de la propia muerte y de la muerte ajena es mayor en personas que presentan puntuaciones más elevadas en autoritarismo de derechas, lo cual podría sustentarse en sus particulares percepciones del mundo como un lugar de por sí amenazante (Campos, 2013). De este modo, el ajuste percibido de ciertas personas se fundamenta en un proceso de sumisión y obediencia a la autoridad, con dosis de convencionalismo, orientación hacia la presión externa, conductas rígidas, estereotipia, amenaza frente al cambio, rechazo de la debilidad, etc. y, como se ha confirmado, ello se relaciona con la saliencia de la amenaza de muerte implícita en los discursos de la autoridad a la que se someten. Se considera que este tipo de recursos que hacen saliente el miedo y la amenaza son fundamentales para la comprensión de buena parte de las teorías de comportamiento sociopolítico actuales y de las acciones que se derivan de las mismas (Rottenbacher \& Schmitz, 2012; Schneider \& Avenburg, 2015; Simancas, 2015).

Como limitaciones del estudio se toman en consideración las propias del diseño transversal de la investigación, así como las derivadas de la selección muestral no 
aleatoria y en el empleo de medidas autoinformadas (sesgo de deseabilidad social), que se intentó contrarrestar mediante la garantía del anonimato y la confidencialidad de los datos. En este sentido, una limitación que podría afectar los resultados se debe a la tendencia de los estudiantes de la muestra a decantarse por una orientación política neutral más que de izquierda o derecha política e, incluso, como muestra de su desinterés político, lo cual podría interpretarse como posible falta de sinceridad en sus respuestas, dada la tendencia de los jóvenes españoles en la expresión de valores, actitudes, proactividad y participación política manifestada en estudios del Instituto de la Juventud en España (Injuve) (Bas, 2014) .

Las líneas futuras de investigación pueden ir orientadas a investigar la influencia del autoritarismo en la conciencia de la muerte y, asimismo, resultaría de interés inducir esta última como paso previo a la evaluación del autoritarismo, con el objetivo de comprobar si situando a los sujetos ante una amenaza potencial de muerte, provoca un mayor número de conductas autoritarias. De hecho, la principal aportación de este estudio es que, contrariamente a la mayoría de las investigaciones previas en las que se relaciona la conducta autoritaria con descriptores sociodemográficos, este se ha centrado en la vinculación con variables actitudinales, en concreto con la conciencia de la muerte, lo cual abre nuevas líneas de investigación en torno a la temática. En la medida en que se conozca mejor esta vinculación específica, así como sus factores asociados, se podrán diseñar y aplicar programas de intervención psicosocial, promoviendo medidas de cambios de actitudes autoritarias en contextos socioeducativos (Garzón, 2012), lo cual lleva a plantear la necesidad de diversificar esta línea de investigación.

\section{Referencias}

Adorno, T. W., Frenkel-Brunswik, E., Levinson, D. J., \& Sanford, R. N. (1950). The authoritarian personality. New York: Harper and Row.

Adorno, T. W., Frenkel-Brunswik, E., Levinson, D. J., \& Sanford, R. N. (1969). The authoritarian personality: Studies in prejudice. New York: Norton.

Ahrens, C. R. \& Innes, J. M. (1994). Attribution of right-wing authoritarianism as a function of self-perceived political opinion. Journal of Social Psychology, 134(3), 383-385. http://dx.doi.org/10.1080/00224545.1994.9711743

Altemeyer, B. (1981). Right-wing authoritarianism. Winnipeg: University of Manitoba Press.

Altemeyer, B. (1996). The authoritarian spectre. Cambridge: Harvard University Press.

Altemeyer, B. (1998). The other authoritarian personality. Advances in Experimental Social Psychology, 30, 47-92.

Bas, J. E. (2014). Educar para innovar, la innovación como cultura: juventud, proactividad, creatividad, participación y visión de futuro compartida. Revista de Estudios de Juventud, 104, 11-30. 
Becker, E. (1973). The denial of death. New York: The Free Press.

Brandt, M. J. \& Henry, P. J. (2012). Gender inequality and gender differences in authoritarianism. Personality and Social Psychology Bulletin, 38(10), 1301-1315. http://dx.doi.org/10.1177/0146167212449871

Bun, J. R., Louiselle, P. A., Misukanis, T. M., \& Mueller, R. A. (1988). Effects of parental authoritarianism and authoritativeness on self-esteem. Personality and Social Psychology Bulletin, 14(2), 271-282.

http://dx.doi.org/10.1177/0146167288142006

Burling, J. W. (1993). Death concerns and symbolic aspects of the self: The effects of mortability salience on status concern and religiosity. Personality and Social Psychology Bulletin, 19(1), 100-105. http://dx.doi.org/10.1177/0146167293191011

Campos, S. (2013). El manejo del terror y su impacto en los motivos de la identidad: un estudio experimental (Tesis doctoral inédita). Pontificia Universidad Católica del Perú, Lima.

Canto, J. M., Perles, F., \& San Martín, J. (2014). El papel del autoritarismo de derechas, del sexismo y de la cultura del honor en la aceptación de los mitos sobre la violación. Revista de Psicología Social: International Journal of Social Psychology, 29(2), 296-318. http://dx.doi.org/10.1080/02134748.2014.918822

Cárdenas, M., Meza, P., Lagues, K., \& Yáñez, S. (2010). Adaptación y validación de la Escala de Orientación a la Dominancia Social (SDO) en una muestra chilena. Universitas Psychologica, 9(1), 161-168. Recuperado de http://www.redalyc.org/articulo.oa?id=64712156013

Cárdenas, M. \& Parra, L. (2010). Adaptación y validación de la versión abreviada de la Escala de Autoritarismos de Derechas (RWA) en una muestra chilena. Revista de Psicología, 19(1), 61-79.

http://dx.doi.org/10.5354/0719-0581.2010.17098

Carvacho, H. \& Haye, A. (2008). Configuración ideológica y estructura social: resucitando el tema desde la psicología política. Revista de Psicología, 17(2), 81-94.

http://dx.doi.org/ 10.5354/0719-0581.2008.17138

Collett, L. -J. \& Lester, D. (1969). The fear of death end the fear of dying. Journal of Psychology, 72(2), 179-81.

http://dx.doi.org/10.1080/00223980.1969.10543496

Comuni, A., Langelotti, L., Jaume, L. C., Rodríguez, F. A., \& Etchezahar, E. (noviembre, 2010). Autoritarismo de derechas y posicionamiento ideológico en estudiantes universitarios. II Congreso Internacional de Investigación y Práctica Profesional en Psicología XVII Jornadas de Investigación Sexto Encuentro de Investigadores en Psicología del Mercosur. Universidad de Buenos Aires. Recuperado de http://www.aacademica.com/000-031/580 
Díaz-Lázaro, C. M. \& Toro, J. (2014). La contribución estadística del autoritarismo, la dominancia social, la empatía y el materialismo a la varianza del prejuicio intergrupal en Puerto Rico. Revista Puertorriqueña de Psicología, 25(1), 118-137.

Duckitt, J. (1989). Authoritarism and group identification: A new view of an old construct. Political Psychology, 10(1), 63-84.

http://dx.doi.org/10.2307/3791588

Duckitt, J. (2000). Culture, personality, and prejudice. En S. Renhson \& J. Duckitt (Eds.), Political psychology: Cultural and crosscultural foundations (pp. 89-106). London: Macmillan.

Duckitt, J. \& Fisher, K. (2003). The impact of social threat on worldview and ideological attitudes. Political Psychology 24(1), 41-74.

http://dx.doi.org/10.1111/0162-895X.00316

Duncan, L. E., Peterson, B. E., \& Winter, D. G. (1997). Authoritarianism and gender roles: Toward a psychological analysis of hegemonic relationships. Personality and Social Psychology Bulletin 23(1), 41-49.

Duriez, B., Van Hiel, A., \& Kossowska, M. (2005). Authoritarianism and social dominance in western and Eastern Europe: The importance of the sociopolitical context and of political interest and involvement. Political Psychology, 26(2), 299-320.

http://dx.doi.org/10.1111/j.1467-9221.2005.00419.x

Ekehammar, B., Akrami, N., Gylje, M., \& Zakrisson, I. (2004). What matters most to prejudice: Basic personality, social dominance orientation or right-wing authoritarianism? European Journal of Personality, 18(6), 463-482.

http://dx.doi.org/10.1002/per.526

Espinosa, A. \& Calderón, A. (2006). Ideología política, valores culturales y miedo a la muerte. Su impacto después de los atentados del 11 de marzo. Psicología Política, 32, 33-58. Recuperado de http://www.uv.es/garzon/psicologia\%20politica/N32-3.pdf

Espinoza, M., Sanhueza, O., \& Barriga, O. (2011). Validación de la Escala de Miedo a la Muerte de Collett-Lester en una muestra de estudiantes de Enfermería. Revista Latinoamericana Enfermagen, 19(5), 1171-1180.

Etchezahar, E. (2012). Las dimensiones del autoritarismo: análisis de la Escala del Autoritarismo del Ala de Derechas (RWA) en una muestra de estudiantes universitarios de la ciudad de Buenos Aires. Psicología Política, 12(25), 591-603. Recuperado de http://pepsic.bvsalud.org/pdf/rpp/v12n25/v12n25a13.pdf

Etchezahar, E. \& Brussino, S. (2015). Dimensiones del autoritarismo, centralidad de la religión y orientaciones religiosas: diferencias en el análisis lineal y no lineal de sus relaciones. Actualidades en Psicología, 29(118), 73-81.

http://dx.doi.org/10.15517/ap.v29i118.18210 
Etchezahar, E., Prado-Gascó, V. J., Jaume, L., \& Brussino, S. (2014). Validación argentina en la Escala de Orientación a la Dominancia Social. Revista Latinoamericana de Psicología, 46(1), 35-43.

http://dx.doi.org/10.1016/S0120-0534(14)70004-4

Farre, B. \& Duckitt, J. (1994). The validity of Tompkins's polarity scale among white South Africans. Journal of Social Psychology, 134(3), 287- 296.

http://dx.doi.org/10.1080/00224545.1994.9711732

Fromm, E. (1941). El miedo a la libertad. Buenos Aires: Gino Germani.

Funke, F. (2005). The dimensionality of right-wing authoritarianism: Lessons from the dilemma between theory and measurement. Political Psychology, 26(2), 195-218.

http://dx.doi.org/10.1111/j.1467-9221.2005.00415.x

Garzón, A. (2012). Cambio de actitudes autoritarias en ambientes educativos. Psicología Política, 45, 61-84. Recuperado de http://www.uv.es/garzon/psicologia\%20politica/N45-4.pdf

Greenberg, J., Pyszczynski, T., \& Solomon, S. (1986). The causes and consequences of the need for self-esteem: A terror management theory. En R. F. Baumeister (Ed.), Public self and private self (pp. 189-212). New York: Springer-Verlag.

http://dx.doi.org/10.1007/978-1-4613-9564-5_10

Greenberg, J., Pyszczynski, T., Solomon, S., Rosenblatt, A., Veeder, M., Kirkland, S., \& Lyon, D. (1990). Evidence for terror management theory: II. The effects of mortality salience reactions to those who threaten or bolster the cultural worldview. Journal of Personality and Social Psychology, 58(2), 308-318.

http://dx.doi.org/10.1037/0022-3514.58.2.308

Greenberg, J., Simon, L., Pyszczynski, T., Solomon, S., \& Chatel, D. (1992). Terror management and tolerance: Does mortality salience always intensify negative reactions to others who threaten one's worldview? Journal of Personality and Social Psychology, 63(2), 212-220.

http://dx.doi.org/10.1037//0022-3514.63.2.212

Greenberg, J., Solomon, S., \& Pyszczynski, T. (1997). Terror management theory of selfesteem and cultural worldviews: Empirical assessments and conceptual refinements. En M. P. Zanna (Ed.), Advances in experimental social psychology (vol. 29, pp. 61-139). San Diego: Academic Press. http://dx.doi.org/10.1016/S0065-2601(08)60016-7

Hernández, M. \& López, P. (2015). Jóvenes españoles vulnerables en tiempos de crisis. Un enfoque multidimensional. Aposta: Revista de Ciencias Sociales, 67, 9-32.

Jackson, L. E. \& Gaertner, L. (2010). Mechanisms of moral disengagement and their differential use by right-wing authoritarianism and social dominance orientation in support of war. Aggressive Behavior, 36(4), 238-250.

http://dx.doi.org/10.1002/ab.20344

Jost, J. T., Glaser, J., Kruglanski, A. W., \& Sulloway, F. J. (2003). Political conservatism as motivated social cognition. Psychological Bulletin, 129(3), 339-375.

http://dx.doi.org/10.1037/0033-2909.129.3.339 
Leidner, B., Castano, E., Zaizer, E., \& Giner-Sorolla, R. (2010). Ingroup glorification, moral disengagement, and justice in the context of collective violence. Personality and Social Psychology Bulletin, 36(8), 1115-1139.

http://dx.doi.org/10.1177/0146167210376391

Manganelli, A. M., Bobbio, A., \& Canova, L. (2007). A short version of the Right-Wing Authoritarianism (RWA) Scale. Personality and Individual Differences, 43(5), 1223-1234. http://dx.doi.org/10.1016/j.paid.2007.03.013

Martin, J. L. (2001). The authoritarian personality, 50 years later: What questions are there for political psychology? Political Psychology, 22(1), 1-26. http://dx.doi.org/10.1111/0162-895X.00223

McClosky, H. \& Chong, D. (1985). Similarities and differences between left-wing and right-wing radicals. British Journal of Political Science, 15(3), 329-363.

http://dx.doi.org/10.1017/S0007123400004221

McFarland, S. G., Ageyev, V. S., \& Djintcharadze, N. (1996). Russian authoritarianism two years after communism. Personality and Social Psychology Bulletin, 22(2), 210-217. http://dx.doi.org/10.1177/0146167296222010

Ovejero, A. (1982). El autoritarismo: enfoque psicológico. El Basilisco, 13, 40-44. Recuperado de http://fgbueno.es/bas/pdf/bas11303.pdf

Ovejero, A. (1986). Diferencias entre los sexos en autoritarismo y dogmatismo. Tabanque, 2, 237-249.

Ovejero, A. (1987). Relación entre los estudios superiores cursados y el autoritarismo, el dogmatismo y el maquiavelismo. Magister: Revista Miscelánea de Investigación, 5, 93111.

Peterson, B. E. \& Zurbriggen, E. L. (2010). Gender, sexuality, and the authoritarian personality. Journal of Personality, 78(6), 1801-1826.

http://dx.doi.org/10.1111/j.1467-6494.2010.00670.x

Petit, L. \& Costa, G. L. (2011). Dominancia social: el género como jerarquía social. Hologramática, 14(3), 71-83. Recuperado de http://www.cienciared.com.ar/ra/usr/3/611/hologramatica_n14_v3pp71_83.pdf

Piñuela, R. (2015). Manejo del terror, ideología política y mecanismos asociados al apoyo de la violencia colectiva: efectos de la amenaza existencial sobre la desconexión moral, la deshumanización y los fundamentos morales (Tesis doctoral inédita). Universidad Complutense de Madrid, Madrid.

Pyszczynski, T., Greenberg, J., \& Solomon, S. (1999). A dual process model of defense against conscious and unconscious death-related thoughts: An extension of terror management theory. Psychological Review, 106(4), 835-845.

http://dx.doi.org/10.1037//0033-295x.106.4.835

Rajan, S. \& Krishnan, V. R. (2002). Impact of gender on influence, power and authoritarianism. Women in Management Review, 17(5), 197-206.

http://dx.doi.org/10.1108/09649420210433157 
Ray, J. J. (1976). Do authoritarians hold authoritarian attitudes? Human Relations, 29(4), 307-325.

http://dx.doi.org/10.1177/001872677602900401

Roccato, M., Re, M., \& Sclauzero, S. (2002). Autoritarismo, nacionalismo y patriotismo. Un estudio con militantes italianos. Psicología Política, 25, 21-35. Recuperado de http://www.uv.es/garzon/psicologia\%20politica/N25-2.pdf

Rokeach, M. (1960). The open and closed mind. New York: Basic Book Inc.

Rottenbacher, J. M. \& Schmitz, M. (2012). Conservadurismo político y tolerancia hacia comportamientos transgresores. Psicología Política, 44, 31-56.

Rubinstein, G. \& Lansisky, L. (2013). Beauty and the best: Authoritarianism and gender roles of Israeli footballers, basketballers, non-athletes, and their wives. Personality and Individual Differences, 55(4), 411-416.

http://dx.doi.org/10.1016/j.paid.2013.03.015

Rosenblatt, A., Greenberg, J., Solomon, S., Pyszczynski, T., \& Lyon, D. (1989). Evidence for terror management theory: I. The effects of mortality salience on reactions to those who violate or uphold cultural values. Journal of Personality and Social Psychology, 57(4), 681-690.

http://dx.doi.org/10.1037/0022-3514.57.4.681

Sarrión, A. (2015). De la ilusión a la desesperanza: se cumplen 30 años de la firma de adhesión de España a la Unión Europea. El siglo de Europa, 1113, 42-44.

Schaffer, B. S. \& Riordan, C. M. (2003). A review of cross-cultural methodologies for organizational research: A best practices approach. Organizational Research Methods, 6(2), 169-215.

http://dx.doi.org/10.1177/1094428103251542

Schneider, C. \& Avenburg, K. (2015). Cultura política: un concepto atravesado por dos enfoques. POSTData: Revista de Reflexión y Análisis Político, 20(1), 109-131. Recuperado de http://www.scielo.org.ar/pdf/postdata/v20n1/v20n1a05.pdf

Schultz, P. W. \& Stone, W. F. (1994). Authoritarianism and Attitudes Toward the Environment. Environment and Behavior, 26(1), 25-37.

http://dx.doi.org/10.1177/0013916594261002

Seoane, G., Arce, C., \& Sabucedo, J. M. (1988). La Escala "Directiveness" de Ray y el autoritarismo. Revista de Psicología Social: International Journal of Social Psychology, $3(1), 71-82$.

http://dx.doi.org/10.1080/02134748.1988.10821575

Seoane, J. \& Garzón, A. (1992). Creencias sociales contemporáneas, autoritarismo y humanismo. Psicología Política, 5, 27-52. Recuperado de http://www.uv.es/garzon/psicologia\%20politica/N5-2.pdf

Shils, E. A. (1954). Authoritarianism: Right and left. En R. Christie \& M. Jahoda (Eds.), Studies in the scope and method of the authoritarian personality (pp. 22-49). Glencoe, Ill.: Free Press. 
Sidanius, J. \& Pratto, F. (1999). Social dominance: An intergroup theory hierarchy and oppression. Cambridge: Cambridge University Press.

Silván-Ferrero, M. P., \& Bustillos, A. (2007). Adaptación de la Escala de Orientación a la Dominancia Social al castellano: validación de la dominancia grupal y la oposición a la igualdad como factores subyacentes. Revista de Psicología Social, 22(1), 3-15.

http://dx.doi.org/10.1174/021347407779697485

Simancas, R. (2015). Democracia, política y el riesgo del "mayoritismo". Temas para el Debate, 242-243, 20-23.

Sirvent, C. (2013). La crisis. Mi crisis. (Infortunio social y consecuencias psiquiátricas). Norte de Salud Mental, 11(45), 111-116. Recuperado de http://antigua.ome-aen.org/norte/45/170x240\%2016\%20La\%20crisis.pdf

Smith, A. \& Winter, D. (2002). Right-wing authoritarianism, party identification and attitudes toward feminism in student evaluations of the Clinton-Lewinsky story. Political Psychology, 23(2), 355-383. http://dx.doi.org/10.1111/0162-895X.00285

Solomon, S., Greenberg, J., \& Pyszczynski, T. (1991). A terror management theory of social behavior: The psychological functions of self-esteem and cultural worldviews. Advances in Experimental Social Psychology, 24, 93-159.

http://dx.doi.org/10.1016/s0065-2601(08)60328-7

SPSS Inc. (2009). Statistics for Windows. Version 18.0.3. Chicago: SPSS Inc.

Stone, W. (2001). Manipulación del terror y autoritarismo. Psicología Política, 23, 7-17. Recuperado de http://www.uv.es/garzon/psicologia\%20politica/N23-1.pdf

Temkin, B. \& Flores-Ivich, G. (2011). Importancia del autoritarismo-dogmatismo en las actitudes socio-políticas. Psicología Política, 43, 65-84. Recuperado de http://www.uv.es/garzon/psicologia\%20politica/N43-3.pdf

Tomás-Sábado, J., Limonero J. T., \& Abdel-Khalek A. M. (2007). Spanish adaptation of the Collett-Lester fear of death scale. Death Studies, 31(3), 249-260. http://dx.doi.org/10.1080/07481180601152625

Ungaretti, J., Etchezahar, E., \& Brussino, S. (2015). La percepción de peligro y competencia como factores predictores del prejuicio hacia diferentes grupos sociales. Escritos de Psicología, 8(1), 30-37.

http://dx.doi.org/10.5231/psy.writ.2015.0912

Velázquez-Gaztelu, J. P. (2015). Un país más pobre y más desigual: la Encuesta de Condiciones de Vida ECV constata el empobrecimiento de la sociedad española durante la crisis. Alternativas Económicas, 27, 26-27.

Walter, M. I. (1996). Personality and emotion: Response to musical mood induction (Tesis de maestría inédita). University of Maine, Orono, EE.UU.

Wolf, M. (2015). La gran crisis: cambios y consecuencias. Lo que hemos aprendido y lo que todavía nos queda por aprender de la crisis financiera. Barcelona: Deusto. 
Wolfe, A. (2005). The authoritarian personality’ revisited. Chronicle of Higher Education, 52(7), 12-13. Recuperado de http://chronicle.com/weekly/v52/i07/07b01201.htm

Zakrisson, I. (2005). Construction of a short version of Right-wing Authoritarianism (RWA) scale. Personality and Individual Differences, 39(5), 863-872. http://dx.doi.org/10.1016/j.paid.2005.02.026

Fecha de recepción: 28 de agosto de 2015 Fecha de aceptación: 14 de octubre de 2015 